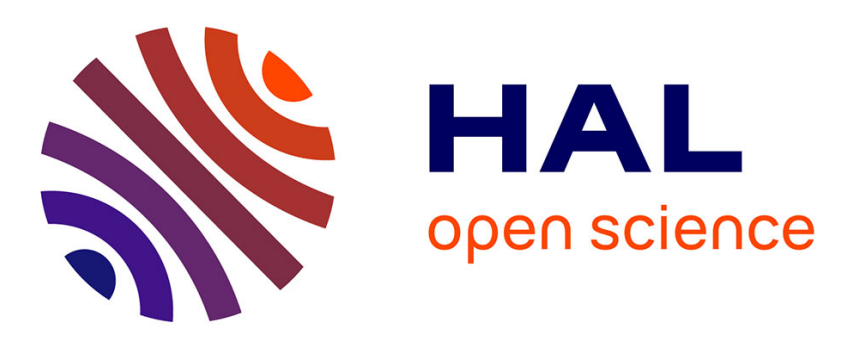

\title{
Self-Assembly of Porphyrin Nanostructures at the Interface Between Two Immiscible Liquids
}

Andrés Molina-Osorio, David Cheung, Colm O'Dwyer, Andrew Stewart, M. Dossot, Grégoire Herzog, Micheál Scanlon

\section{- To cite this version:}

Andrés Molina-Osorio, David Cheung, Colm O'Dwyer, Andrew Stewart, M. Dossot, et al.. SelfAssembly of Porphyrin Nanostructures at the Interface Between Two Immiscible Liquids. Journal of Physical Chemistry C, 2020, 10.1021/acs.jpcc.0c00437 . hal-02502377

\section{HAL Id: hal-02502377 \\ https://hal.univ-lorraine.fr/hal-02502377}

Submitted on 2 Dec 2020

HAL is a multi-disciplinary open access archive for the deposit and dissemination of scientific research documents, whether they are published or not. The documents may come from teaching and research institutions in France or abroad, or from public or private research centers.
L'archive ouverte pluridisciplinaire HAL, est destinée au dépôt et à la diffusion de documents scientifiques de niveau recherche, publiés ou non, émanant des établissements d'enseignement et de recherche français ou étrangers, des laboratoires publics ou privés. 


\section{Self-assembly of Porphyrin Nanostructures at the Interface between Two Immiscible Liquids}

Andrés F. Molina-Osorio, ${ }^{1}$ David Cheung, ${ }^{2}$ Colm O'Dwyer, ${ }^{3,4}$ Andrew A. Stewart, ${ }^{5}$ Manuel

Dossot, ${ }^{6}$ Grégoire Herzog, ${ }^{6}$ and Micheál D. Scanlon. ${ }^{1,4, *}$

${ }^{1}$ The Bernal Institute and Department of Chemical Sciences, School of Natural Sciences, University of Limerick (UL), Limerick V94 T9PX, Ireland.

${ }^{2}$ School of Chemistry, National University of Ireland, Galway, University Road, Galway, Ireland.

${ }^{3}$ School of Chemistry, and Tyndall National Institute, University College Cork, Cork, T12 YN60 Ireland.

${ }^{4}$ Advanced Materials and Bioengineering Research (AMBER) centre.

${ }^{5}$ The Bernal Institute and Department of Physics, School of Natural Sciences, University of Limerick (UL), Limerick V94 T9PX, Ireland.

${ }^{6}$ CNRS-Université de Lorraine, LCPME UMR 7564, 405 Rue de Vandoeuvre, 54600 Villers-lèsNancy, France.

This document is a postprint. Final version has been published in Journal of Physical Chemistry C, 2020, 124, 6929-6937 (https://pubs.acs.org/doi/10.1021/acs.jpcc.0c00437) 
ABSTRACT. One of the many evolved functions of photosynthetic organisms is to synthesize light harvesting nanostructures from photoactive molecules such as porphyrins. Engineering synthetic analogues with optimized molecular order necessary for the efficient capture and harvest of light energy remains challenging. Here, we address this challenge by reporting the self-assembly of zinc(II) meso-tetrakis(4-carboxyphenyl)porphyrins into films of highly ordered nanostructures. The self-assembly process takes place selectively at the interface between two immiscible liquids (water|organic solvent), with kinetically stable interfacial nanostructures formed only at $\mathrm{pH}$ values close to the $\mathrm{p} K \mathrm{a}$ of the carboxyphenyl groups. Molecular dynamics simulations suggest that the assembly process is driven by an interplay between the hydrophobicity gradient at the interface and hydrogen bonding in the formed nanostructure. Ex situ XRD analysis and in situ UV/vis and steady state fluorescence indicates the formation of chlathrate type nanostructures that retain the emission properties of their monomeric constituents. The self-assembly method presented here avoids the use of acidic conditions, additives such as surfactants and external stimuli, offering an alternative for the realization of light-harvesting antennas in artificial photosynthesis technologies.

\section{INTRODUCTION}

Photosynthetic organisms universally exploit antenna systems to capture high energy photons and funnel this excitation energy toward a coupled reaction centre. ${ }^{1,2}$ Self-assembled molecular antennae consisting of multi-layers of chromophores, such as porphyrins, can potentially function as high efficiency light harvesters due to their exceptionally high molar absorption coefficients $\left(10^{5} \mathrm{~cm}^{-1} \cdot \mathrm{M}^{-1}\right) .^{3}$ However, to mimic the evolved efficiency of these antennas and avoid “concentration quenching" of the excited state at disordered trap sites, ${ }^{3}$ the supramolecular packing of the individual chromophores must be precisely controlled and show long-range molecular order. $^{4,5}$ 
Molecular self-assemblies at "soft" liquid|air or immiscible liquid|liquid interfaces can exhibit the required macroscale long-range order. ${ }^{6}$ These soft interfaces are exceptionally smooth, and have no inherent defects leading to an unrivalled macroscale uniformity in molecule-interface interactions. ${ }^{78}$ By contrast, the grain boundaries, step defects and edge sites always present at solid|liquid interfaces can impede diffusion of adsorbed molecules, trapping them in local energy minima as molecules stick to defect sites. ${ }^{9,10}$ The uniform templating of adsorbed molecules at liquid|air or immiscible liquid|liquid interfaces has been exploited to create a variety of porphyrin assemblies, ${ }^{11-16}$ yet a facile and robust route to create highly-ordered porphyrin nanostructures remains challenging.

Here we describe the self-assembly of light harvesting nanostructures from readily available, water-soluble and symmetrically substituted porphyrins at mild $\mathrm{pH}$ conditions. Thus, we avoid acidic conditions (that would lead to the expulsion of the central metal ion), ${ }^{17}$ as well as the use of synthetically challenging amphiphilic porphyrin molecules, ${ }^{18}$ and more complicated routes using additives (e.g., divalent cations or surfactants) ${ }^{19-21}$ or external stimuli (e.g., electric fields). ${ }^{22-24} \mathrm{We}$ chose zinc(II) meso-tetrakis(4-carboxyphenyl)porphyrin (ZnTPPc) as a prototypical model system to demonstrate this new means of self-assembly at an immiscible aqueous|organic interface. Simply contacting aqueous ZnTPPc solutions prepared in citrate buffer at $\mathrm{pH}$ values between 5.1 and 6.0 with a neat, immiscible organic phase of $\alpha, \alpha, \alpha$-trifluorotoluene (TFT) lead to the immediate formation of films of porphyrin nanostructures. We rationalise our findings using molecular dynamics (MD) simulations that highlights the key role of the hydrophobicity gradient at the immiscible aqueous|organic interface, and carboxylic acid-carboxylate hydrogen bonding interactions in the formed nanostructure. The latter were found to be maximised at $\mathrm{pH}$ values close to the $\mathrm{pKa}$ of the carboxylic groups within the nanostructure $(\mathrm{pH} 5.8),{ }^{25}$ leading to kinetically 
stable interfacial nanostructures. The presence of multi-layers with strong visible light absorption, and the crystalline macroscale long-range molecular order in the porphyrin nanostructure, suggests these films as promising light-harvesting antennae in artificial photosynthetic technologies.

\section{EXPERIMENTAL SECTION}

2.1. Chemicals. All reagents were used as received without further purification. Mesotetrakis(4-carboxyphenyl)porphyrin $\left(\mathrm{H}_{2} \mathrm{TPPc}, \geq 98 \%\right.$ ) and its zinc(II) derivative (ZnTPPc, $\geq 98 \%$ ) were obtained from Porphychem. Lithium hydroxide ( $\mathrm{LiOH}, \geq 98 \%$ ), citric acid $\left(\mathrm{H}_{3} \mathrm{Cit}, \geq 99.5 \%\right.$ ), decamethylferrocene (97\%), and 1,2-dichloroethane (DCE, $\geq 99.0 \%$ ) were purchased from SigmaAldrich, and $\alpha, \alpha, \alpha$-trifluorotoluene (TFT, $\geq 99 \%$ ) from Acros Organics. All aqueous solutions were prepared using Milli-Q ${ }^{\circledR}$ deionized water $(18.2 \mathrm{M} \Omega \cdot \mathrm{cm})$. Aqueous solutions of ZnTPPc were prepared by directly dissolving the solid in the lithium citrate buffer pre-adjusted to the desired $\mathrm{pH}$, followed by sonication of the solution for three minutes. Initially, $\mathrm{H}_{2} \mathrm{TPPc}$ was insoluble in the buffer at neutral $\mathrm{pH}$. Therefore, the solid was dissolved first in $\mathrm{LiOH}$ and the $\mathrm{pH}$ subsequently adjusted with $\mathrm{H}_{3}$ Cit. The ionic strength of each lithium citrate buffer solution containing either porphyrin was maintained at $10( \pm 2) \mathrm{mM}$. For the photoelectrochemistry experiments, bis(triphenylphosphoranylidene) ammonium chloride $(\mathrm{BACl}, \quad 97 \%)$ and lithium tetrakis(pentafluorophenyl)borate diethyletherate $\left(\left[\mathrm{Li}\left(\mathrm{OEt}_{2}\right)\right] \mathrm{TB}\right)$ were obtained from Sigma$\begin{array}{llll}\text { Aldrich and } & \text { Boulder }\end{array}$ Bis(triphenylphosphoranylidene)ammonium tetrakis(pentafluorophenyl)borate (BATB) was prepared by metathesis of $\mathrm{BACl}$ and $\left.\mathrm{Li}\left(\mathrm{OEt}_{2}\right)\right] \mathrm{TB}$, as described previously. ${ }^{26}$

2.2. Characterisation methodology. The films of Por-INs were gently transferred to a silicon substrate for SEM, XRD and Raman characterisation, or an Agar Scientific TEM grid (Holey Carbon film $300 \mathrm{Mesh} \mathrm{Cu}$ ) for TEM imaging, by bringing the solid supports into contact with the 
interface. Prior to imaging, the samples were sequentially rinsed with water and TFT, and dried under a stream of nitrogen gas. TEM images were acquired on a Thermo Fisher double aberration corrected Titan Themis, spot size 6, on a Gatan Oneview detector. SEM images were obtained on a FEI Quanta 650 FEG high resolution SEM. X-ray diffraction patterns (XRD) were acquired in - $-2 \theta$ geometry with a Phillips Xpert PW3719 diffractometer using $\mathrm{Cu} \mathrm{K} \alpha$ radiation $(\lambda=0.15418$ $\mathrm{nm}$, operation voltage $40 \mathrm{kV}$, current $40 \mathrm{~mA}$ ). Patterns were also acquired in $\omega-2 \theta$ geometry using a PANalytical X'pert PRO XRD. UV/vis absorbance spectra were collected in a Thermo Scientific Evolution 60S UV/vis spectrophotometer with illumination provided by a Xenon light source (accuracy $\pm 0.8 \mathrm{~nm}$ ). Steady-state fluorescence experiments were performed in a LS 55 Perkin Elmer Fluorescence spectrometer. The experimental configuration implemented to obtain UV/vis and steady-state fluorescence spectra of the Por-INs is illustrated in Figure S1. Raman measurements were carried out using a Horiba Jobin Yvon T64000 spectrometer equipped with a nitrogen cooled CCD detector. The laser wavelength was $532 \mathrm{~nm}$ with a power of $13 \mathrm{~mW}$. Raman spectra were obtained in 10 acquisitions of 30 seconds duration.

2.3. Adsorption isotherms. Vials containing biphasic systems of ZnTPPc or $\mathrm{H}_{2} \mathrm{TPP}$ in lithium citrate buffer (10 mM ionic strength, $\mathrm{pH} 5.8)$ at different initial bulk concentrations and TFT as the organic phase were prepared and left to stand for 24 hours. After this time, the Por-INs formed and all remaining unadsorbed porphyrin in the bulk aqueous phase was extracted by thoroughly rinsing with porphyrin-free buffer solution. The solutions containing unadsorbed porphyrin were collected and analysed by UV/vis absorbance spectroscopy to quantify the porphyrin concentration therein (final bulk concentration). By subtracting the final from the initial bulk concentrations, the surface concentration (number of moles adsorbed per geometric area of aqueous|organic interface) was determined. All quantifications were performed using a calibration curve. 
2.4. Molecular dynamics simulations. Simulations of interfacial adsorption and assembly were performed using a pre-equilibrated water|TFT interface, consisting water and TFT regions with 4000 water molecules and 1226 TFT molecules, respectively. Full details are provided in the Supporting Information.

\subsection{Photocurrent transient measurements at an electrified aqueous|organic interface.}

Photocurrent measurements with a DC illumination were performed using the LED driver provided by Metrohm Autolab in conjunction with a PGSTAT204 in a 4-electrode configuration as presented in Figure S2. The overlap of the emission spectrum of the LED with the absorption spectra of ZnTPPc, $\mathrm{H}_{2}$ TPPc and their respective Por-INs is presented in Figure S3. The determination of the photon flux at the electrified liquid|liquid interface as a function of the LED driving current is outlined in Figure S4.

\section{RESULTS AND DISCUSSION}

3.1. Triggering the formation of interfacial nanostructures. The selective formation of ZnTPPc nanostructures at the interface between water and TFT was observed upon contacting a ZnTPPc aqueous solution with neat TFT. A yellow/green colour was observed at the water|TFT interface within minutes, easily distinguishable from the purple colour of the bulk ZnTPPc aqueous solution, and associated with the formation of porphyrin interfacial nanostructures (Por-INs) (Figure 1a). Self-assembly was observed only at $\mathrm{pH}$ values where the ratio between neutral, protonated $\left(\mathrm{H}_{4}[\mathrm{ZnTPPc}]\right)$ and tetra-anionic, deprotonated $\left([\mathrm{ZnTPPc}]^{4-}\right)$ species was close to 1 $\left(\mathrm{pH}=\mathrm{pKa} \mathrm{aOOH}^{\mathrm{CO}}=5.8\right)$, see Figure S5. Mono-, di- and triprotonated ZnTPPc species may also be present. However, only one $\mathrm{pKa}$ was observed for the potentiometric titration of ZnTPPc with hydrochloric acid (Figure S6) suggesting that the different $\mathrm{pKa}$ values of the carboxylic groups fall 
within a very narrow interval of $\mathrm{pH}$. Nevertheless, it must be noted that the interfacial protonation equilibria is complex. Despite using a buffered aqueous electrolyte, an implicit assumption cannot be made that the $\mathrm{pH}$ at the liquid|liquid boundary, where nanostructure formation is initiated, is the same as in the bulk. Numerous studies have shown that local interfacial $\mathrm{pH}$ values can be several units different from the bulk aqueous phase. ${ }^{27-29}$ Furthermore, the $\mathrm{pKa}$ of the carboxyphenyl groups may differ from their bulk values, observed previously for other acid/base equilibria at the water|air interface..$^{30,31}$

MD simulations show that the hydrophilic, anionic $[\mathrm{ZnTPPc}]^{4-}$ species prefer to sit in water at a distance of $\sim 3-5 \AA$ above the interface due to their hydrophilic nature (Figure 1b), and suggest that initially, hydrophobic neutral $\mathrm{H}_{4}[\mathrm{ZnTPPc}]$ species accumulate at the interface driven by the hydrophobicity gradient at the immiscible liquid|liquid interface (Figure 1b and Figure S7). This interfacial layer acts as a template structure for the $[\mathrm{ZnTPPc}]^{4-}$ species to adsorb via carboxylic acid-carboxylate hydrogen-bonding and $\pi-\pi$ interactions. In this manner, a crystalline film of ZnTPPc nanostructures builds up layer-by-layer at the interface. Akin to the clathrate crystals of ZnTPPc developed by Goldberg and coworkers, ${ }^{32-36}$ the strength of each individual hydrogen bond or $\pi-\pi$ interaction may be insubstantial, but the cooperative effect allows the net enthalpies of these multivalent interactions to cumulatively rival the strength of a covalent bond and stabilise the PorIN. Ex situ scanning electron microscopy (SEM) images reveal the thickness of the film of ZnPorINs to be approximately $135( \pm 5) \mathrm{nm}$ (Figure 1c). 

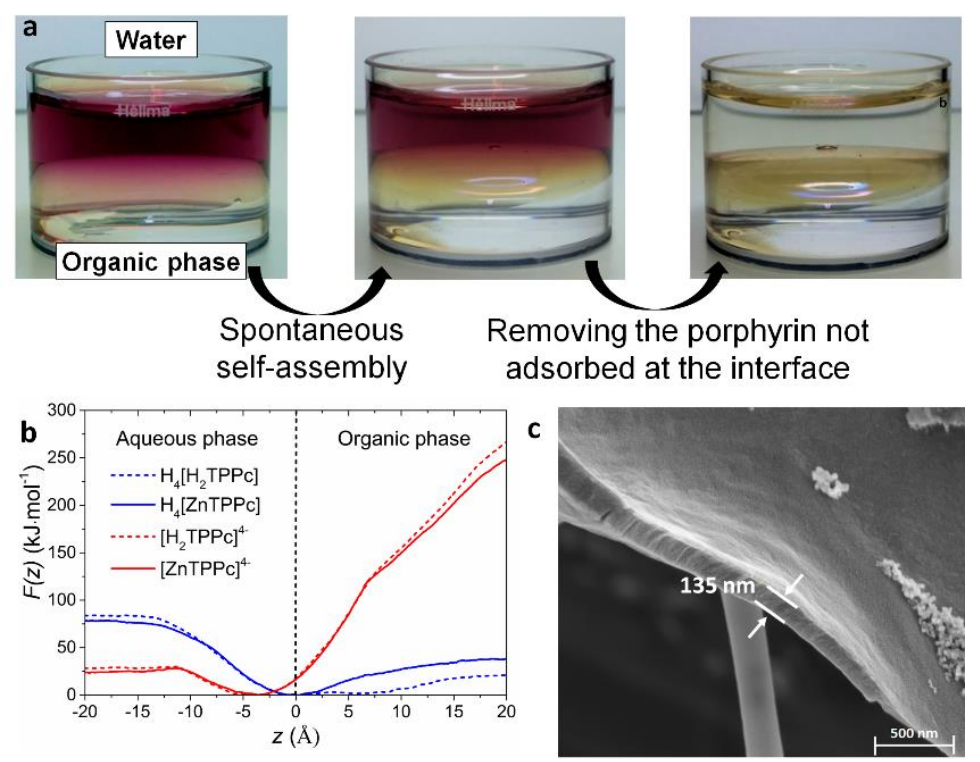

d
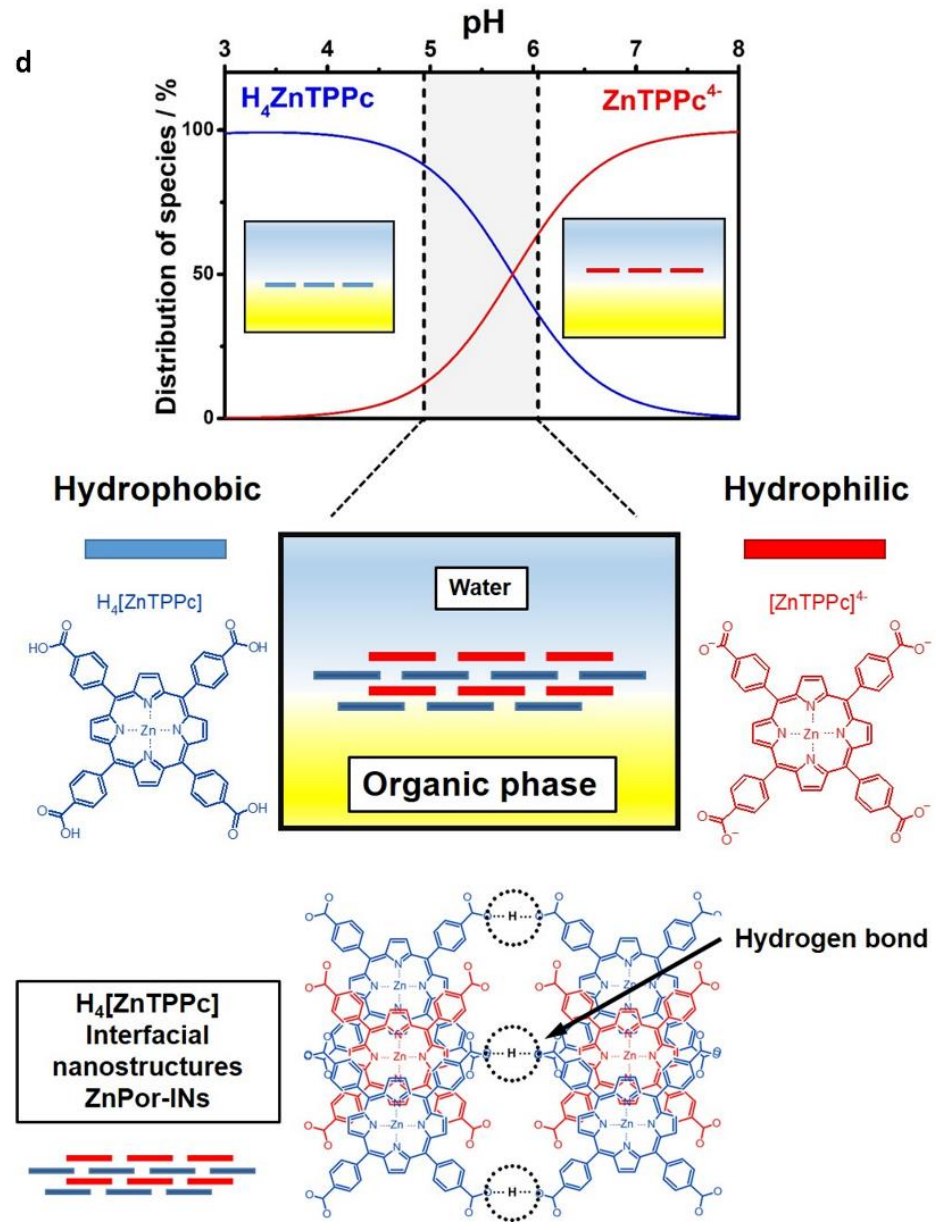

Figure 1. Formation of porphyrin interfacial nanostructures. a) Optical images of the formation of Por-INs at the interface between water and TFT. b) Computed potential of mean force or free energy profiles for translation of ZnTPPc molecules across the water|TFT interface, averaged over 
$10 \mathrm{~ns}$ of free molecular dynamics, with the carboxylate groups on the 4-carboxyphenylsubstitutents either fully deprotonated, [ZnTPPc] ${ }^{4}$ (solid red line) or fully protonated $\mathrm{H}_{4}[\mathrm{ZnTPPc}]$ (solid blue line). c) Scanning electron microscopy (SEM) image of a film of ZnPor-INs transferred from the water|TFT interface to a copper grid with a holey carbon substrate. d) Distribution percentage of neutral $\mathrm{H}_{4}[\mathrm{ZnTPP}]$ (blue line) and anionic [ZnTPPc] ${ }^{4-}$ (red line) porphyrin species in the aqueous phase as a function of $\mathrm{pH}$, with a schematic description of the layered structure of the ZnPor-INs, and chlathrate structure of ZnPor-INs obtained from ex situ XRD analysis (vide infra). Note, for clarity, only a simple hydrogen bonding model is displayed for the chlathrate structure of the ZnPor-INs. However, coordination of carboxyl groups to the central zinc atom is also important to form a well ordered 3D-structure, as described vide infra.

Control experiments and MD simulations demonstrated that ZnTPPc and free-base $\mathrm{H}_{2} \mathrm{TPPc}$ molecules are kinetically stable in solution at $\mathrm{pH} 5.8$, and do not undergo spontaneous bulk aggregation in the concentration range studied (Figure S8). This indicates that the Por-INs form only by self-assembly in situ at the water|TFT interface. Thus, to achieve selective Por-IN formation, the $\mathrm{pH}$ of the aqueous solution must be controlled across a narrow $\mathrm{pH}$ range between 5.1 and 6.0 (Figure 1d). More alkaline conditions inhibit formation of the Por-INs due to electrostatic repulsion between tetra-anionic porphyrins. More acidic conditions induce the spontaneous formation of aggregates in the bulk aqueous phase (Figure S9). This $\mathrm{pH}$ dependency of Por-IN formation indicates that cooperative H-bonding is key for self-assembly of the nanostructure. This finding is in line with a detailed examination of the photoelectrochemistry of ZnTPPc and decamethylferrocene at the water|1,2-dichloroethane (DCE) interface by Girault and co-workers. ${ }^{37}$ Based on the photocurrent dependence on the angle of polarisation under total internal reflection, they concluded that the spontaneous 2D coverage of ZnTPPc molecules at the interface is $\mathrm{pH}$ dependent, sharply decreasing above $\mathrm{pH}$ 6. While no interfacial nanostructures were observed by Girault and co-workers, ${ }^{37}$ their data strongly correlates with our observations 
that spontaneous 2D assembly is the initial step in the generation of 3D interfacial nanostructures. Furthermore, nanostructure self-assembly is not restricted to a single immiscible biphasic system, with experiments showing that Por-INs also form selectively at the water|DCE interface (Figure S10).

3.2. Molecular structure of the Por-INs. Transmission electron microscopy (TEM) and X-ray diffraction (XRD) analysis confirm the crystalline and layered nature of the porphyrin interfacial nanostructures. Ex situ TEM images with corresponding selected area electron diffraction (SAED) analysis, and patterns from ZnPor-INs (Figure 2a-c) were acquired after immobilization of the film on an amorphous hydrophilized glass substrate. The diffraction pattern for ZnPor-INs bears a striking resemblance to NAFS (nanofilm on a solid surface) structural models for liquid phase interfacial synthesis of highly ordered molecular nanosheets (Figure $2 \mathrm{a}$ ). ${ }^{38}$ SAED and XRD estimate a $0.55-0.57 \mathrm{~nm}$ interplanar spacing between $\{110\}$ planes, with a $\sim 1.1 \mathrm{~nm}$ periodicity measured from HRTEM lattice fringes. The intense 220 reflection is consistent with a lack of layer-on-layer stacking order (due to non-registered stacking), but a highly crystalline nanosheet material is formed. The (200) reflections indicate strong axial coordination that is a distinct feature of layering along the c-axis normal to the support. Clearly discernible (hk0) reflections are consistent with a tetragonal unit cell with preferred growth orientation along the plane of the liquid|liquid interface (Figure 2b,c). Experiments with nanostructures of the free base $\mathrm{H}_{2} \mathrm{TPPc}$ $\left(\mathrm{H}_{2}\right.$ Por-INs) demonstrate the key role of the central metal ion in enhancing the crystallinity of the film of porphyrin interfacial nanostructures. $\mathrm{H}_{2}$ Por-INs grown in the same way also form a crystalline 2D layered material, and show a similar XRD pattern but with a more pronounced amorphous background and suppressed reflection intensity compared to the ZnPor-INs (Figure 2d,e). A schematic representation of the crystalline and interdigitated layered clathrate-type 
structure of the ZnPor-INs is illustrated in Figure 2f. This structure assumes interdigitation to account for the reduced unit cell spacing compared to a NAFS-1 or NAFS-2 tetragonal metalorganic nanosheet crystalline structure.
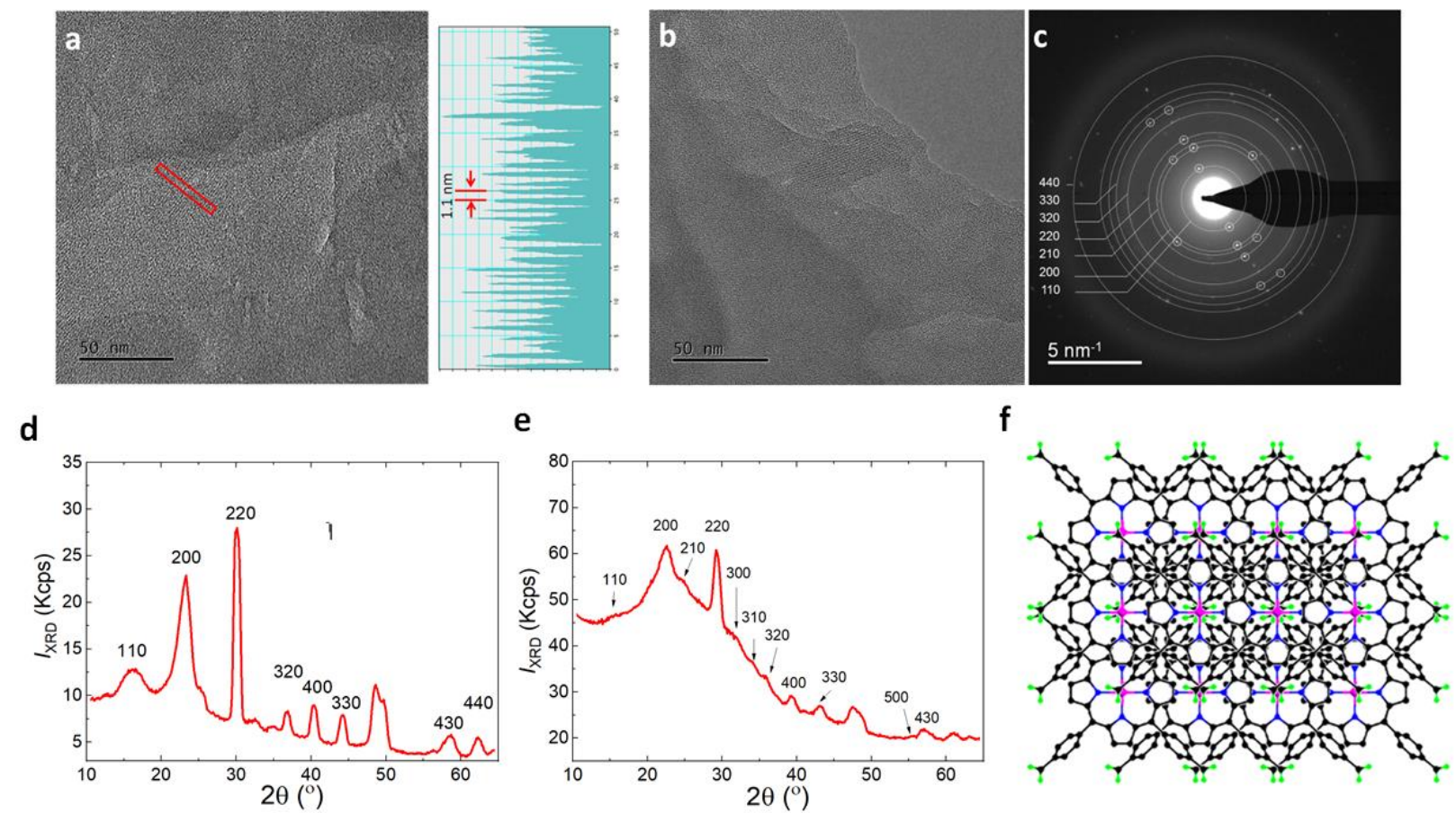

Figure 2. Characterisation of ZnPor-INs and $\mathrm{H}_{2}$ Por-INs ex situ by transmission electron microscopy (TEM) and X-ray diffraction (XRD). a) TEM image of ZnPor-INs transferred from the water|TFT interface to a copper grid with a holey carbon substrate, taken at $185 \mathrm{k}$ magnification. The profile of the lattice fringing shown on the right is taken from the area marked by the red box in the TEM image. b) TEM image of ZnPor-INs, taken at $185 \mathrm{k}$ magnification at an accelerating voltage of $300 \mathrm{kV}$ and c) the corresponding diffraction pattern with a $40 \mu \mathrm{m}$ selected area aperture and camera length of $580 \mathrm{~mm}$. d) XRD pattern of the ZnPor-INs transferred to a silicon substrate. Patterns were acquired in $\omega-2 \theta$ geometry and clearly discernible $(h k 0)$ reflections confirms a tetragonal unit cell and dominant $c$-axis layering. e) XRD of the $\mathrm{H}_{2}$ Por-INs. f) Schematic representation of the crystalline and layered clathrate-type structure of the ZnPorINs. Black balls represent carbon, pink is zinc, blue are nitrogen, and green are oxygen. 


\subsection{The interfacial nanostructures represent a kinetically trapped metastable state rather}

than a thermodynamically stable state. The interfacial concentration of Por-INs was measured as a function of the solution concentration of ZnTPPc and $\mathrm{H}_{2} \mathrm{TPPc}$, respectively. Over the concentration range studied (0.5-100 $\mu \mathrm{M})$, ZnTPPc adsorption followed a Brunauer-Emmet-Teller (BET) isotherm behaviour, whereas $\mathrm{H}_{2} \mathrm{TPPc}$ adsorption followed a linear isotherm behaviour (Figure 3). Using the BET model for liquid phase adsorption reported by Ebadi et al., ${ }^{39}$ the isotherm obtained for ZnTPPc adsorption was fit to Eqn. 1:

$$
\Gamma=\Gamma_{m} \frac{K_{1} C_{e q .}}{\left(1-K_{2} C_{\text {eq. }}\right)\left(1-K_{2} C_{e q}+K_{1} C_{e q .}\right)}
$$

in which $K_{1}$ and $K_{2}$ are the pseudo-equilibrium constants of adsorption to form the first and second layers of the ZnPor-INs, $\Gamma$ and $\Gamma_{m}$ are the equilibrium and monolayer porphyrin surface concentrations, respectively, and $C_{e q}$. is the equilibrium concentration of the porphyrin in solution. Non-linear fitting determined $\Gamma_{m}$ as $13.08( \pm 0.94) \mathrm{nmol} \cdot \mathrm{cm}^{-2}$, and $K_{1}$ and $K_{2}$ as $4.55 \times 10^{-2}( \pm 0.25)$ and $8.79 \times 10^{-3}\left( \pm 2.21 \times 10^{-4}\right) \mathrm{L} \cdot \mu \mathrm{mol}$, respectively ( $95 \%$ confidence). Thus, the Gibbs free energy of adsorption $\left(\Delta \mathrm{G}_{\mathrm{ads}}\right)$ of $\mathrm{ZnTPPc}$ molecules to form the first and second layers of the ZnPor-INs are $7.65 \mathrm{~kJ} \cdot \mathrm{mol}^{-1}$ and $11.72 \mathrm{~kJ} \cdot \mathrm{mol}^{-1}$, respectively. These values suggest that the interfacial nanostructures represent a kinetically trapped metastable state rather than a thermodynamically stable state. In other words, the interfacial molecular self-assembly process is under kinetic control, and such out-of-equilibrium self-assembly systems are known to yield porphyrin nanostructures that are inaccessible through the spontaneous thermodynamic process. ${ }^{40}$ The latter kinetic control has also been observed in supramolecular polymerization of porphyrins, ${ }^{41}$ and the self-assembly of polyelectrolytes, ${ }^{42}$ perylenes,${ }^{43}$ and other types of nanostructures. ${ }^{44}$ 


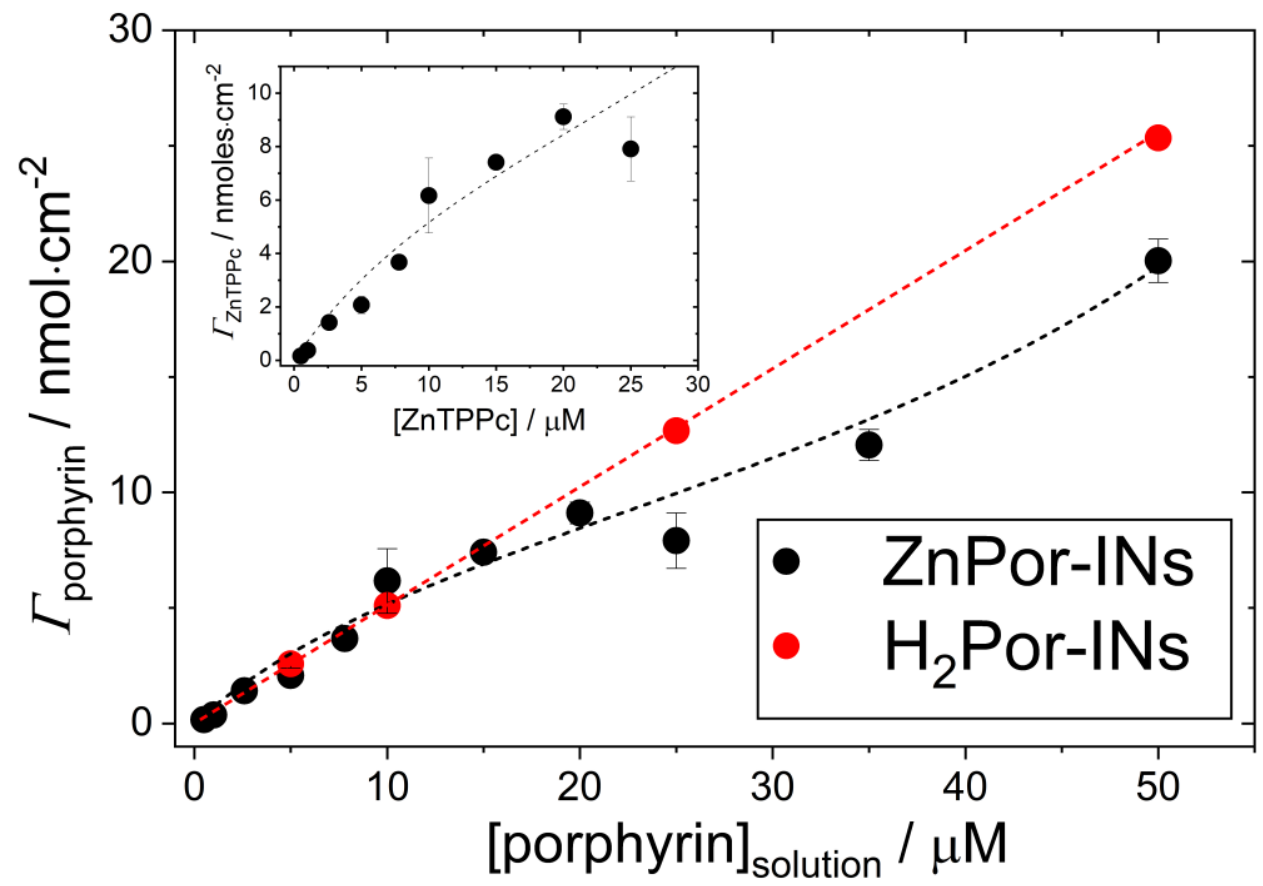

Figure 3 Adsorption isotherms obtained at $20{ }^{\circ} \mathrm{C}$ of $\mathrm{ZnTPPc}$ and $\mathrm{H}_{2} \mathrm{TPPc}$ at the water|TFT interface. Inset: Adsorption isotherm data for ZnTPPc at the water|TFT interface, highlighting the Brunauer-Emmet-Teller (BET) behavior

The difference in the adsorption isotherms for $\mathrm{ZnTPPc}$ and $\mathrm{H}_{2}$ TPPc further emphasises the influence of the metal centre during porphyrin interfacial adsorption. Previous studies at an aqueous|dodecane interface demonstrated that the adsorption of an oil-soluble metalloporphyrin was highly dependent on the nature of the central metal ion due to the different water coordination geometries around different metals. ${ }^{45}$ Also, the study by Girault and co-workers ${ }^{37}$ noted vide supra, concluded that there is a direct link between the surface coverage and orientation of ZnTPPc adsorbed at the interface. A dihedral angle between the porphyrin ring and the surface normal in the range of 60 to $75^{\circ}$ was observed, depending on the bulk ZnTPPc concentration and the application of a Galvani potential difference at the liquid|liquid interface, and rationalized in terms of minimizing the contact of deprotonated carboxyl groups to the hydrophobic phase. ${ }^{37}$ We used 
MD simulations to quantify the influence of the presence or absence of $\mathrm{Zn}^{2+}$, and the protonation state, on the adsorption of ZnTPPc and $\mathrm{H}_{2}$ TPPc at the water|TFT interface. For all model variants,

there is a significant degree of orientational freedom (Figure S11), with all molecules adopting a range of orientations relative to the interface. This is reflected in the orientational angle probability distributions which have peaks at a low $\left(<15^{\circ}\right)$ angle but remain non-zero for all angles. (Figure S11). This suggests that the difference in the adsorption behaviour stems from different supramolecular packing modes within the Por-INs and not their interaction with the liquid|liquid interface.

\subsection{Influence of nanostructure formation on the photophysical properties of the assembly.}

In situ UV/vis absorption spectra of ZnTPPc in solution and ZnPor-INs are shown in Figure 4a and Figure S12. The $\lambda_{\max }$ value of the Soret band for the ZnPor-INs is blue-shifted (from 422 to $413 \mathrm{~nm}$ with respect to ZnTPPc in solution). Perturbation of the electronic absorption spectra in terms of shifts and broadening of the Soret band, indicate the presence of multiple structural domains within the ZnPor-INs. Analogous to ZnTPPc in solution, ZnPor-INs display two Q bands, indicating that the porphyrin ring remains metallated with $\mathrm{Zn}^{2+}$ and thus retains the $D_{4 \mathrm{~h}}$ symmetry. Additionally, in solution, ZnTPPc presented one main peak centred at $422 \mathrm{~nm}$ (Figure 4a and Table S1). This peak further indicates the monomeric nature of ZnTPPc under the experimental conditions used during this work. The latter is supported with the adherence of ZnTPPc in solution to the Beer-Lambert law across an extensive concentration range (Figure S12), and with previous studies. ${ }^{46}$ 
a

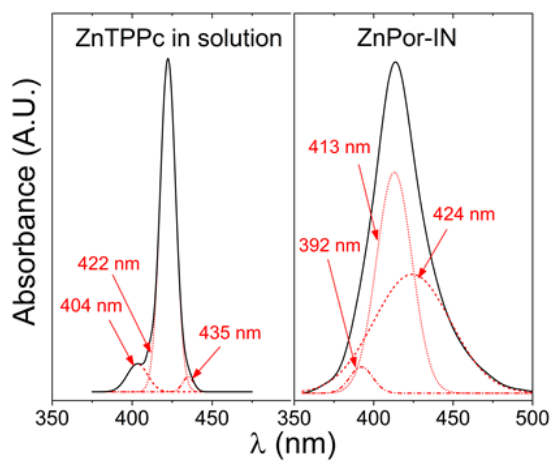

b

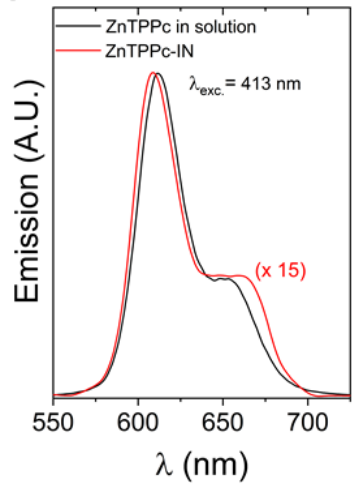

C

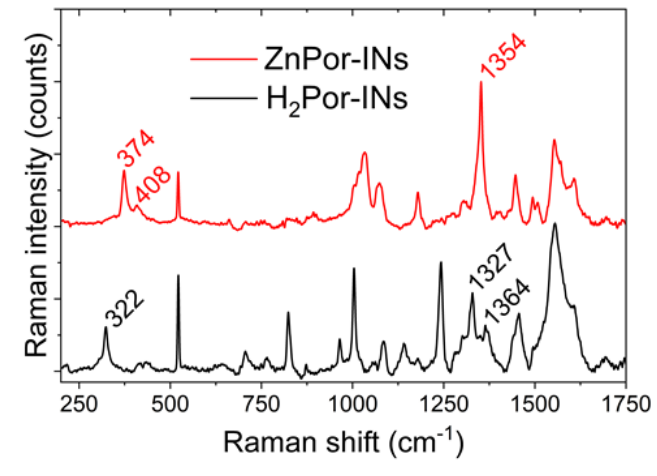

Figure 4. Spectroscopic characterisation of the ZnPor-INs by in situ UV/vis absorbance and steady-state fluorescence, and ex situ Raman spectroscopy. Deconvolution of the Soret absorbance bands for a) ZnTPPc in solution and ZnPor-INs. b) Comparison of the fluorescence emission spectra of ZnTPPc in solution and ZnPor-INs for an excitation wavelength $\left(\lambda_{\text {exc. }}\right)$ of $418 \mathrm{~nm}$. c) Comparison of the Raman spectra of films of ZnPor- and $\mathrm{H}_{2}$ Por-INs transferred from the water|TFT interface to a silicon substrate (see Table S2 for peak assignments).

In situ steady-state fluorescence spectra was measured for an excitation wavelength $\left(\lambda_{\text {exc. }}\right)$ of 413 $\mathrm{nm}$ (Figure 4b). Comparison of the emission profiles of ZnTPPc in solution and the ZnPor-INs shows that the $\mathrm{Q}(0,0)$ transient is slightly blue-shifted and $\mathrm{Q}(0,1)$ transition slightly red-shifted upon nanostructure formation. Following Kasha's exciton model, the ZnPor-INs can be classified

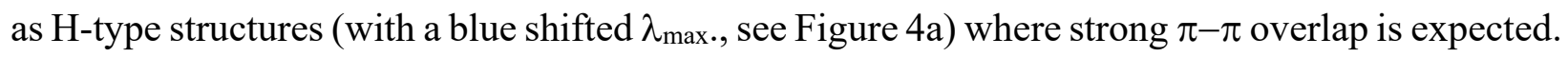
$\pi-\pi$ interactions are reported to lower exciton diffusion and fluorescence rates. ${ }^{47,48}$ However, energy transfer within these types of molecular assemblies is also affected by other parameters such as intermolecular distance, molecular packing orientation or molecular crystallinity. ${ }^{49}$ The importance of the latter is highlighted in several works where high carrier mobility is observed in structures with strong $\pi-\pi$ stacking interactions. ${ }^{49,50}$ For example, Vohra et al. ${ }^{50}$ reported crystalline lamellar structures of thiophene polymers with short $\pi-\pi$ stacking distance as highly 
efficient active layers in solar cells. Thus, the retention of fluorescence emission after nanostructure formation demonstrates how the long-range molecular order in the nanostructure leads to diminished concentration quenching, a key attribute when designing light-harvesting antennae in artificial photosynthetic technologies. Additional dependence of the emission properties of these nanostructures on the excitation wavelength is presented in Figures S13 and S14.

Since $\mathrm{ZnTPPc}$ self-assembles in mild $\mathrm{pH}$ conditions, expulsion of $\mathrm{Zn}^{2+}$ was avoided, as confirmed by analysis of the effect of nanostructure formation on the vibrational modes of the ZnPor- and $\mathrm{H}_{2}$ Por-INs by ex situ Raman spectroscopy (Figure 4c). Prominent differences between the two are entirely consistent with previous comparisons of metallo- and free-base 4carboxyphenyl-substituted porphyrin Raman spectra (Table S2). ${ }^{51,52}$ The retention of $\mathrm{Zn}^{2+}$ is a key advantage of the interfacial self-assembly method described over common aggregation methods in bulk solution at acidic conditions. The presence of the metal increases the inter-system crossing (ISC) rate constant, $k_{\text {ISC, }}$ due to the heavy atom effect, increasing the probability of the forbidden $\mathrm{S}_{1} \rightarrow \mathrm{T}_{1}$ transition. From the $\mathrm{T}_{1}$ state, relaxation may occur via phosphorescence or charge transfer. The long-lived (up to millisecond) excited triplet state lifetimes provides sufficient time for the excited state to efficiently interact with ground state quencher molecules. ${ }^{53}$

As a proof-of-concept, the photoactivity of the ZnPor-INs was demonstrated by mediating interfacial photo-induced electron transfer (PET) between redox species chemically confined to different sides of a liquid|liquid interface using the methodology pioneered by Girault and coworkers (Figure 5a). ${ }^{37,53-55}$ The multi-layers of porphyrin in the ZnPor-INs floating at the interface function as light harvesters. Upon illumination, the generated triplet excited state was reductively quenched by electron transfer from decamethylferrocene, and the ground state regenerated by hole 
transfer from $\mathrm{O}_{2}$. The charge separation was accompanied by an electrical photocurrent through an external circuit, and the latter increased on application of a more positive Galvani potential difference (Figure 5b-c).

Control cyclic voltammograms (CVs) were carried out, in the dark and with chopped LED illumination, in the presence of the ZnPor-INs at the interface and decamethylferrocene in the TFT phase (Figure S15). Charge transfer peaks due to the adsorbed ZnPor-INs precluded the study of applied Galvani potentials less than $-0.1 \mathrm{~V}$ (Figure S15). Decamethylferrocene is a relatively strong electron donor, capable of reducing dissolved $\mathrm{O}_{2}$, and consequently leading to a dark current at positive Galvani potentials with an onset of $\sim+0.15 \mathrm{~V}$. The mechanism underlying this dark current has been identified previously as the reduction of $\mathrm{O}_{2}$ by first proton transfer to the organic phase, followed by formation of a decamethylferrocene-hydride. The latter then reacts with dissolved $\mathrm{O}_{2}$ in the TFT to generate a peroxyl radical species, $\mathrm{HO}_{2} \cdot{ }^{\cdot 56}$ However, as seen in Figure S15, the photocurrents obtained are superimposed upon this dark current and can be resolved by background subtraction of the dark current (as is the case for the photocurrent transients shown in Figures $5 \mathrm{~b}$ and $\mathrm{c})$.

A major future perspective outside the scope of this article is an in-depth exploration of PET at electrified liquid|liquid interfaces using this photoactive film. In particular, a detailed analysis on the potential dependence of the magnitude and line-shape of the photocurrent transient measurements is required and will lead to an elegantly simple system to realise a new approach to artificial photosynthesis entirely based on a self-assembled system. 

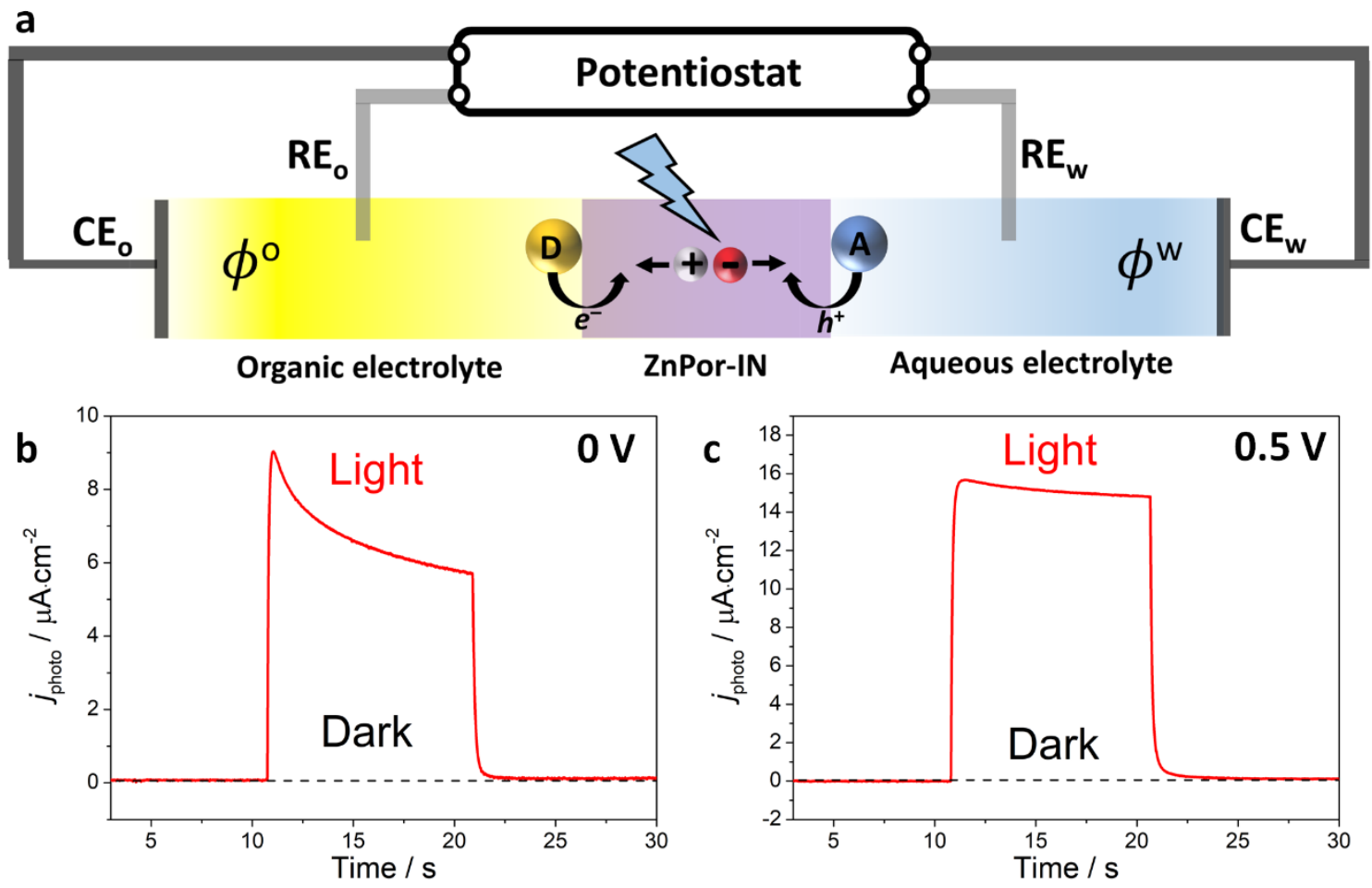

Figure 5. Photoconversion at the interface between two immiscible liquids. a) Schematic of "softphotoconversion"; converting light energy to chemical energy using dye-sensitised electrified liquid|liquid interfaces. The donor species (D) is decamethylferrocene and the acceptor species (A) is $\mathrm{O}_{2}$. The $\mathrm{ZnTPPc}$ triplet excited state in the crystalline, layered $\mathrm{ZnPor}-\mathrm{INs}$ undergoes reductive quenching by decamethylferrocene, with $\mathrm{O}_{2}$ regenerating the ground state. Thus, light energy is converted to chemical energy in the form of the oxidised donor $\left(\mathrm{D}^{+}\right)$and reduced acceptor $\left(\mathrm{A}^{-}\right)$ spatially separated on either side of the water|TFT interface. Photocurrent transients were measured $\mathbf{b})$ at $0 \mathrm{~V}$ and $\mathbf{c})$ at a positive polarisation of the interface $(0.5 \mathrm{~V})$, at $\mathrm{pH} 5.8$ and $5 \mathrm{mM}$ decamethyferrocene in the TFT phase. The ZnPor-INs floating at the water|TFT interface were illuminated with an LED $\left(470 \mathrm{~nm}\right.$ at $\left.50 \mathrm{~mW} \cdot \mathrm{cm}^{-2}\right)$ controlled by the potentiostat. The water|TFT interface was electrified using a specialised 4-electrode electrochemical cell $\left(\mathrm{CE}_{\mathrm{w}}\right.$ and $\mathrm{CE}_{\mathrm{o}}$ are the counter electrodes in the water and TFT phases, respectively, and $\mathrm{RE}_{\mathrm{w}}$ and $\mathrm{RE}_{\mathrm{o}}$ are the reference 
electrodes in each phase). The supporting organic electrolyte was $5 \mathrm{mM}$ bis(triphenylphosphoranylidene)ammonium tetrakis(pentafluorophenyl)borate (BATB).

\section{CONCLUSIONS}

The defect-free nature of the water|organic interface provides an ideal platform to self-assemble interfacial nanostructures with unique structural arrangements. In this Article, we report the selfassembly of interfacial nanostructures of zinc(II) meso-tetrakis(4-carboxyphenyl)porphyrin. The nanostructures are stabilised by cooperative hydrogen bonding and, due to the templating interaction of the interface with adsorbed porphyrin molecules, possess a highly ordered structure. This approach uniquely harnesses the difference in hydrophobicity between the neutral protonated and tetra-anionic non-protonated versions of the porphyrin at $\mathrm{pKa}$ conditions, combined with the introduction of a hydrophobicity gradient to trigger interfacial self-assembly. We open a new avenue to kinetically stable porphyrin nanostructure formation under mild experimental conditions without the need for acidic $\mathrm{pH}$, designer amphiphilic porphyrin molecules, aggregation-inducing additives or external triggers. The feasibility of using such nanostructures for light collection and harvesting was demonstrated in situ by measuring photocurrents associated with interfacial photoinduced electron transfer across the water|TFT interface.

\section{ASSOCIATED CONTENT}

\section{Supporting Information}

Supporting experimental methods (in situ microscopy, molecular dynamic simulations, photocurrent transient measurements), methodology of porphyrin interfacial nanostructure 
formation, potentiometric titration data, molecular dynamic studies of porphyrins in the bulk (dimerisation free energy calculations) or at the liquid|liquid interface (mechanism of layer-bylayer formation of the porphyrin interfacial nanostructures, determining probability distributions of the angle of orientation of adsorbed porphyrins), spectroscopic studies of the porphyrins in bulk aqueous solutions (influence concentration and $\mathrm{pH}$ on dimerisation or aggregation, respectively), in situ spectroscopic studies of porphyrin interfacial nanostructures (UV/vis, fluorescence and Raman).

\section{AUTHOR INFORMATION}

\section{Corresponding Author}

*micheal.scanlon@ul.ie (M.D. Scanlon)

\section{ORCID ID numbers}

Andrés F. Molina-Osorio: 0000-0001-8356-6381

David L. Cheung: 0000-0002-3994-2295

Colm O’Dwyer: 0000-0001-7429-015X

Andrew Stewart: 0000-0002-3081-5644

Manuel Dossot: 0000-0003-0575-025X

Grégoire Herzog: 0000-0003-1932-9300

Micheál D. Scanlon: 0000-0001-7951-7085

\section{Author Contributions}

The manuscript was written through contributions of all authors. All authors have given approval to the final version of the manuscript. 


\section{ACKNOWLEDGMENTS}

This publication has emanated from research by M.D.S. and A.F.M.-O. supported by the European Research Council through a Starting Grant (agreement no. 716792) and in part by a research grant from Science Foundation Ireland (SFI) (grant number 13/SIRG/2137). M.D.S. and A.F.M.-O. acknowledge funding through Irish Research Council New Foundations Awards (2014 and 2015) to facilitate the research. A.M.O., M.D.S., G.H. and M.D. are grateful to the support of the Irish Research Council and Campus France for travel support between the French and Irish groups through their joint ULYSSES programme. G. H. is grateful to the French Programme Investissement d'Avenir (PIA) “Lorraine Université d'Excellence” (Reference No. ANR-15IDEX-04-LUE) for the partial financial support of this work. C.O.D. acknowledges support from Science Foundation Ireland (SFI) under Grant Numbers 13/TIDA/E2761, 14/IA/2581 and 15/TIDA/2893. Computational facilities and support for the molecular dynamics simulations were provided by the Irish Centre for High-End Computing (ICHEC). Ivan Robayo-Molina (University of Limerick) is acknowledged for assistance in carrying out the potentiometric titrations.

\section{REFERENCES}

(1) Scholes, G. D.; Fleming, G. R.; Olaya-Castro, A.; Van Grondelle, R. Lessons from Nature about Solar Light Harvesting. Nat. Chem. 2011, 3 (10), 763-774.

(2) Mirkovic, T.; Ostroumov, E. E.; Anna, J. M.; Van Grondelle, R.; Govindjee; Scholes, G. D. Light Absorption and Energy Transfer in the Antenna Complexes of Photosynthetic Organisms. Chem. Rev. 2017, 117 (2), 249-293.

(3) Otsuki, J. Supramolecular Approach towards Light-Harvesting Materials Based on 
Porphyrins and Chlorophylls. J. Mater. Chem. A 2018, 6 (16), 6710-6753.

(4) Magna, G.; Monti, D.; Di Natale, C.; Paolesse, R.; Stefanelli, M. The Assembly of Porphyrin Systems in Well-Defined Nanostructures: An Update. Molecules 2019, 24 (23), 4307.

(5) Medforth, C. J.; Wang, Z.; Martin, K. E.; Song, Y.; Jacobsen, J. L.; Shelnutt, J. A. SelfAssembled Porphyrin Nanostructures. Chem. Commun. 2009, 7345 (47), 7261-7277.

(6) Bigioni, T. P.; Lin, X. M.; Nguyen, T. T.; Corwin, E. I.; Witten, T. A.; Jaeger, H. M. Kinetically Driven Self Assembly of Highly Ordered Nanoparticle Monolayers. Nat. Mater. 2006, 5 (4), 265-270.

(7) Michael, D.; Benjamin, I. Molecular Dynamics Simulation of the Water|nitrobenzene Interface. J. Electroanal. Chem. 1998, 450 (2), 335-345.

(8) Strutwolf, J.; Barker, A. L.; Gonsalves, M.; Caruana, D. J.; Unwin, P. R.; Williams, D. E.; Webster, J. R. P. Probing Liquid|liquid Interfaces Using Neutron Reflection Measurements and Scanning Electrochemical Microscopy. J. Electroanal. Chem. 2000, 483 (1), 163-173.

(9) Mendes, A. C.; Baran, E. T.; Reis, R. L.; Azevedo, H. S. Self-Assembly in Nature: Using the Principles of Nature to Create Complex Nanobiomaterials. WIREs Nanomed Nanobiotechnol 2013, 5 (6), 582-612.

(10) Grzybowski, B. A.; Wilmer, C. E.; Kim, J.; Browne, K. P.; Bishop, K. J. M. Self-Assembly: From Crystals to Cells. Soft Matter 2009, 5 (6), 1110-1128.

(11) Rong, Y.; Chen, P.; Wang, D.; Liu, M. Porphyrin Assemblies through the Air/water Interface: Effect of Hydrogen Bond, Thermal Annealing, and Amplification of 
Supramolecular Chirality. Langmuir 2012, 28 (15), 6356-6363.

(12) Ponce, C. P.; Araghi, H. Y.; Joshi, N. K.; Steer, R. P.; Paige, M. F. Spectroscopic and Structural Studies of a Surface Active Porphyrin in Solution and in Langmuir-Blodgett Films. Langmuir 2015, 31 (50), 13590-13599.

(13) Babu, S. S.; Bonifazi, D. Self-Organization of Polar Porphyrinoids. Chempluschem 2014, 79 (7), 895-906.

(14) Numata, M.; Kinoshita, D.; Taniguchi, N.; Tamiaki, H.; Ohta, A. Self-Assembly of Amphiphilic Molecules in Droplet Compartments: An Approach toward Discrete Submicrometer-Sized One-Dimensional Structures. Angew. Chemie - Int. Ed. 2012, 51 (8), $1844-1848$.

(15) Xie, F.; Zhuo, C.; Hu, C.; Liu, M. H. Evolution of Nanoflowers and Nanospheres of Zinc Bisporphyrinate Tweezers at the Air/water Interface. Langmuir 2017, 33 (15), 3694-3701.

(16) Qiu, Y.; Chen, P.; Liu, M. Interfacial Assemblies of Atypical Amphiphilic Porphyrins: Hydrophobicity/hydrophilicity of Substituents, Annealing Effects, and Supramolecular Chirality. Langmuir 2010, 26 (19), 15272-15277.

(17) Nagatani, H.; Watarai, H. Direct Spectrophotometric Measurement of Demetalation Kinetics of 5,10,15,20-tetraphenylporphyrinatozinc(II) at the Liquid-liquid Interface by a Centrifugal Liquid Membrane Method. Anal. Chem. 1998, 70 (14), 2860-2865.

(18) Lin, L.; Wang, T.; Lu, Z.; Liu, M.; Guo, Y. In Situ Measurement of the Supramolecular Chirality in the Langmuir Monolayers of Achiral Porphyrins at the Air/aqueous Interface by Second Harmonic Generation Linear Dichroism. J. Phys. Chem. C 2014, 118 (13), 6726- 
6733.

(19) Qian, D. J.; Nakamura, C.; Miyake, J. Multiporphyrin Array from Interfacial MetalMediated Assembly and Its Langmuir-Blodgett Films. Langmuir 2000, 16 (24), 9615-9619.

(20) Cheung, D. L.; Carbone, P. How Stable Are Amphiphilic Dendrimers at the Liquid-liquid Interface? Soft Matter 2013, 9 (29), 6841-6850.

(21) Guo, P.; Zhao, G.; Chen, P.; Lei, B.; Jiang, L.; Zhang, H.; Hu, W.; Liu, M. Porphyrin Nanoassemblies via Surfactant-Assisted Assembly and Single Nanofiber Nanoelectronic Sensors for High-Performance H2O2 Vapor Sensing. ACS Nano 2014, 8 (4), 3402-3411.

(22) Nagatani, H.; Samec, Z.; Brevet, P.-F.; Fermín, D. J.; Girault, H. H. Adsorption and Aggregation of Meso-tetrakis(4-Carboxyphenyl)porphyrinato Zinc(II) at the Polarized water|1,2-Dichloroethane Interface. J. Phys. Chem. B 2003, 107 (3), 786-790.

(23) Yamamoto, S.; Nagatani, H.; Imura, H. Potential-Induced Aggregation of Anionic Porphyrins at Liquid|liquid Interfaces. Langmuir 2017, 33 (39), 10134-10142.

(24) Yamamoto, S.; Nagatani, H.; Morita, K.; Imura, H. Potential-Dependent Adsorption and Orientation of Meso- Substituted Porphyrins at Liquid|liquid Interfaces Studied by Polarization-Modulation Total Internal Reflection Fluorescence Spectroscopy. J. Phys. Chem. C 2016, 120 (13), 7248-7255.

(25) Maiti, N. C.; Mazumdar, S.; Periasamy, N. J- and H-Aggregates of Porphyrin-Surfactant Complexes: Time-Resolved Fluorescence and Other Spectroscopic Studies †. J. Phys. Chem. B 1998, 102 (97), 1528-1538.

(26) Suárez-Herrera, M. F.; Scanlon, M. D. On the Non-Ideal Behaviour of Polarised Liquid- 
Liquid Interfaces. Electrochim. Acta 2019, 328, 1-9.

(27) Zhao, X.; Ong, S.; Wang, H.; Eisenthal, K. B. New Method for Determination of Surface pKa Using Second Harmonic Generation. Chem. Phys. Lett. 1993, 214 (2), 203-207.

(28) Xiao, X. D.; Vogel, V.; Shen, Y. R. Probing the Proton Excess at Interfaces by Second Harmonic Generation. Chem. Phys. Lett. 1989, 163 (6), 555-559.

(29) Castro, A.; Bhattacharyya, K.; Eisenthal, K. B. Energetics of Adsorption of Neutral and Charged Molecules at the Air/water Interface by Second Harmonic Generation: Hydrophobic and Solvation Effects. J. Chem. Phys. 1991, 95 (2), 1310-1315.

(30) Eisenthal, K. B. Liquid Interfaces Probed by Second-Harmonic and Sum-Frequency Spectroscopy. Chem. Rev. 1996, 96 (4), 1343-1360.

(31) Tamburello-Luca, A. A.; Hébert, P.; Antoine, R.; Brevet, P. F.; Girault, H. H. Optical Surface Second Harmonic Generation Study of the Two Acid/base Equilibria of Eosin B at the Air/water Interface. Langmuir 1997, 13 (16), 4428-4434.

(32) Diskin-Posner, Y.; Goldberg, I. From Porphyrin Sponges to Porphyrin Sieves: A Unique Crystalline Lattice of Aquazinc tetra(4-Carboxyphenyl)porphyrin with Nanosized Channels. Chem. Commun. 1999, 1961-1962.

(33) Diskin-Posner, Y.; Patra, G. K.; Goldberg, I. Crystal Engineering of 2-D and 3-D Multiporphyrin Architectures - The Versatile Topologies of TetracarboxyphenylporphyrinBased Materials. Eur. J. Inorg. Chem. 2001, 2001 (10), 2515-2523.

(34) Diskin-Posner, Y.; Dahal, S.; Goldberg, I. New Effective Synthons for Supramolecular SelfAssembly of Meso-Carboxyphenylporphyrins. Chem. Commun. 2000, 585-586. 
(35) George, S.; Goldberg, I. Self-Assembly of Supramolecular Porphyrin Arrays by Hydrogen Bonding: New Structures and Reflections. Cryst. Growth Des. 2006, 6 (3), 755-762.

(36) Goldberg, I. Crystal Engineering of Metalloporphyrin Molecular Sieves and Zeolite Analogues. Acta Crystallogr. Sect. A Found. Crystallogr. 2000, 56 (7), s122-s122.

(37) Jensen, H.; Kakkassery, J. J.; Nagatani, H.; Fermín, D. J.; Girault, H. H. Photoinduced Electron Transfer at Liquid|liquid Interfaces. Part IV. Orientation and Reactivity of Zinc tetra(4-Carboxyphenyl) Porphyrin Self-Assembled at the water|1,2-Dichloroethane Junction. J. Am. Chem. Soc. 2000, 122 (44), 10943-10948.

(38) Motoyama, S.; Makiura, R.; Sakata, O.; Kitagawa, H. Highly Crystalline Nanofilm by Layering of Porphyrin Metal-Organic Framework Sheets. J. Am. Chem. Soc. 2011, 133 (15), $5640-5643$.

(39) Ebadi, A.; Soltan Mohammadzadeh, J. S.; Khudiev, A. What Is the Correct Form of BET Isotherm for Modeling Liquid Phase Adsorption? Adsorption 2009, 15 (1), 65-73.

(40) Fukui, T.; Kawai, S.; Fujinuma, S.; Matsushita, Y.; Yasuda, T.; Sakurai, T.; Seki, S.; Takeuchi, M.; Sugiyasu, K. Control over Differentiation of a Metastable Supramolecular Assembly in One and Two Dimensions. Nat. Chem. 2017, 9 (5), 493-499.

(41) Ogi, S.; Sugiyasu, K.; Manna, S.; Samitsu, S.; Takeuchi, M. Living Supramolecular Polymerization Realized through a Biomimetic Approach. Nat. Chem. 2014, 6 (3), 188195.

(42) Wu, H.; Ting, J. M.; Werba, O.; Meng, S.; Tirrell, M. V. Non-Equilibrium Phenomena and Kinetic Pathways in Self-Assembled Polyelectrolyte Complexes. J. Chem. Phys. 2018, 149 
(16).

(43) Tidhar, Y.; Weissman, H.; Wolf, S. G.; Gulino, A.; Rybtchinski, B. Pathway-Dependent Self-Assembly of Perylene Diimide/peptide Conjugates in Aqueous Medium. Chem. - A Eur. J. 2011, 17 (22), 6068-6075.

(44) Yan, Y.; Huang, J.; Tang, B. Z. Kinetic Trapping-a Strategy for Directing the SelfAssembly of Unique Functional Nanostructures. Chem. Commun. 2016, 52 (80), 1187011884.

(45) Nagatani, H.; Watarai, H. Specific Adsorption of Metal Complexes of Tetraphenylporphyrin at Dodecane-Water Interface. Chem. Lett. 1997, 26 (2), 167-168.

(46) Pasternack, R. F.; Francesconi, L.; Raff, D.; Spiro, E. Aggregation of nickel(II), copper(II), and zinc(II) Derivatives of Water-Soluble Porphyrins. Inorg. Chem. 1973, 12 (11), 26062611.

(47) Huijser, A.; Savenije, T. J.; Kroeze, J. E.; Siebbeles, L. D. A. Exciton Diffusion and Interfacial Charge Separation in Meso- tetraphenylporphyrin/TiO2 Bilayers: Effect of Ethyl Substituents. J. Phys. Chem. B 2005, 109 (43), 20166-20173.

(48) Verma, S.; Ghosh, H. N. Exciton Energy and Charge Transfer in Porphyrin Aggregate/semiconductor (TiO2) Composites. J. Phys. Chem. Lett. 2012, 3 (14), 18771884.

(49) Ya-Rui, S.; Hui-Ling, W.; Ya-Ting, S.; Yu-Fang, L. Theoretical Study of the Charge Transport Mechanism in $\pi$-Stacked Systems of Organic Semiconductor Crystals. CrystEngComm 2017, 19 (40), 6008-6019. 
(50) Vohra, V.; Kawashima, K.; Kakara, T.; Koganezawa, T.; Osaka, I.; Takimiya, K.; Murata, H. Efficient Inverted Polymer Solar Cells Employing Favourable Molecular Orientation. Nat. Photonics 2015, 9 (6), 403-408.

(51) Vlčková, B.; Matějka, P.; Šimonová, J.; Čermáková, K.; Pančoška, P.; Baumruk, V. Surface-Enhanced Resonance Raman Spectra of Free Base 5,10,15,20-tetrakis(4Carboxyphenyl)porphyrin and Its Silver Complex in Systems with Silver Colloid: Direct Adsorption in Comparison to Adsorption via Molecular Spacer. J. Phys. Chem. 1993, 97 (38), 9719-9729.

(52) Cotton, T. M.; Schultz, S. G.; Van Duyne, R. P. Surface-Enhanced Resonance Raman Scattering from Water-Soluble Porphyrins Adsorbed on a Silver Electrode. J. Am. Chem. Soc. 1982, 104 (24), 6528-6532.

(53) Fermín, D. J.; Dung Duong, H.; Ding, Z.; Brevet, P.-F.; Girault, H. H. Photoinduced Electron Transfer at Liquid/liquid Interfaces Part II. A Study of the Electron Transfer and Recombination Dynamics by Intensity Modulated Photocurrent Spectroscopy (IMPS). Phys. Chem. Chem. Phys. 1999, 1 (7), 1461-1467.

(54) Fermín, D. J.; Duong, H. D.; Ding, Z.; Brevet, P. F.; Girault, H. H. Photoinduced Electron Transfer at Liquid/liquid Interfaces. Part III. Photoelectrochemical Responses Involving Porphyrin Ion Pairs. J. Am. Chem. Soc. 1999, 121 (43), 10203-10210.

(55) Fermín, D. J.; Ding, Z.; Duong, H. D.; Brevet, P.-F.; Girault, H. H. Photoinduced Electron Transfer at Liquid/Liquid Interfaces. 1. Photocurrent Measurements Associated with Heterogeneous Quenching of Zinc Porphyrins. J. Phys. Chem. B 1998, 102 (50), 1033410341. 
(56) Jane Stockmann, T.; Deng, H.; Peljo, P.; Kontturi, K.; Opallo, M.; Girault, H. H. Mechanism of Oxygen Reduction by Metallocenes near Liquid|liquid Interfaces. J. Electroanal. Chem. 2014, 729, 43-52. 


\section{TOC graphic}

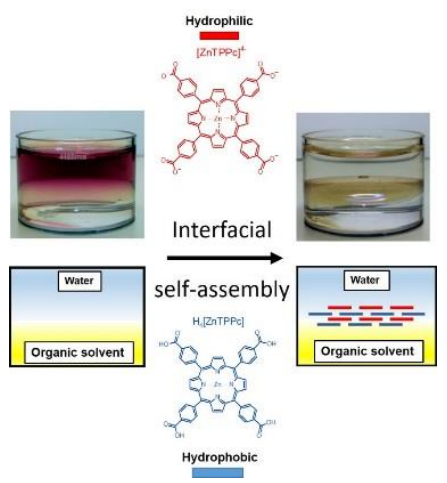

$\mathrm{Zn}$ porphyrins self-assemble at the interface between two immiscible liquids driven by a hydrophobicity gradient and hydrogen bond interactions. 